\title{
Propuesta metodológica para el diseño de intersecciones urbanas
}

Sandra Uribe

Recibido 11 de junio de 2009. Aprobado el 23 de octubre de 2009

\section{Resumen}

Los diseños geométricos de carreteras y vías urbanas son un amplio y diverso campo de acción para los actuales diseñadores, puesto que este trabajo demanda, no sólo los factores matemáticos propios del diseño como pueden ser las restricciones en las tres dimensiones (diseño horizontal, diseño vertical y transversal), sino también el impacto social y ambiental que éste pueda generar, mediante la alteración del espacio público. El objeto del diseño es mejorar el entorno y las condiciones de circulación vehicular, de acuerdo con las necesidades viales proyectadas y en cumplimiento de las normas de diseño que señala el Instituto Nacional de Vías (INVIAS).

El uso de software representa una gran ventaja para el diseño; sin embargo es evidente la ausencia de normas específicas para la planeación de vías urbanas en Colombia, que le brinden al diseñador, herramientas para cada caso particular. Además en el mercado actual, no existe ningún software que tenga implícitos los parámetros y límites que indican las normas aplicables, por lo cuál, estos programas finalmente se convierten en tan solo una herramienta que permite agilizar cálculos.

\section{Palabras clave:}

Diseño geométrico de vías, intersecciones viales.

\begin{abstract}
Highway, local roads and streets geometric designs are a diverse and challenging field for designers nowadays, not only because of the characteristic mathematical demands of every design (such us the three dimensional constrains: horizontal, vertical and cross-sectional alignments) but also the environmental and social impacts that the design might generate, by altering the public space. The objective of the a design is to improve the environment and traffic conditions, according to foreseen road demands and always in compliance with the design standards specified by INVIAS (National Roads Institute).

Currently the use of customized software's is an advantage for the design phase, however there is a lack of specific regulations and practices for an adequate planning of urban roads in Colombia which provide the designer with suitable designing tools for each particular case. Even more in today's highly technological market there is not a program or software that engages the regulations currently in force. Ultimately these programs have only become a technical aid to expedite mathematical calculations. In the end the rightful application of design features is in the hands of a sound designer.
\end{abstract}

\section{Keywords}

Ways geometric design, road intersections. 


\section{Introducción}

Las intersecciones viales ofrecen un potencial de investigación en diseño geométrico de vías, sobre todo en una ciudad con tantas limitantes como Bogotá. La capital colombiana es una ciudad con aproximadamente 9 millones de habitantes que diariamente se enfrentan a un problema como el tráfico. Se cuenta con la capacidad, la topografía, los conocimientos, las condiciones para mejorar la malla vial y se cuenta también con la necesidad de disponer de un manual de diseños de intersecciones para afrontar la solución de la actual problemática.

Si se compara la magnitud de las intersecciones viales de Bogotá con otras capitales latinoamericanas, el atraso resulta evidente. Hace aproximadamente 40 años Caracas (Venezuela) contaba con mejor disposición vial que la que existe en la actualidad en Bogotá.

Aunque el escenario económico es determinante en este factor, el nivel ingenieril de Intersecciones como "La araña" y "El pulpo" marcan una gran diferencia.

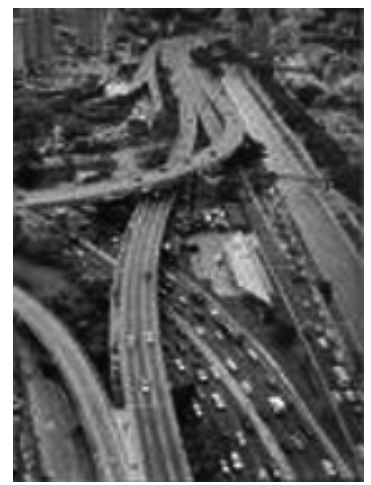

Figura 1. Distribuidor vial - Caracas. Fuente: El autor

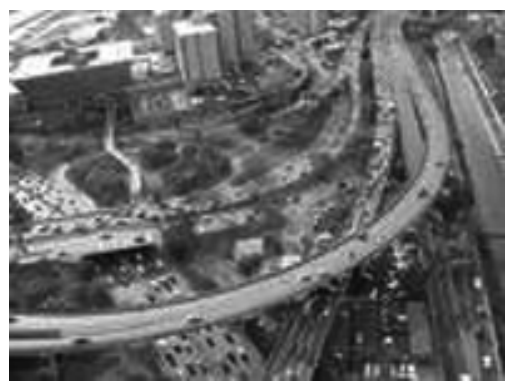

Figura 2. Distribuidor vial - Caracas. Fuente: El autor

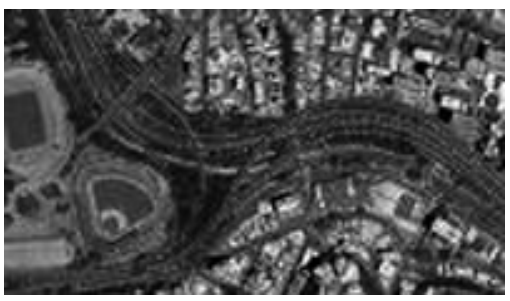

Figura 3. Distribuidor vial - El Pulpo. Fuente: El autor
En los últimos años, la demanda vial ha crecido por el aumento del parque automotor. Se puede decir que la oferta de infraestructura vial es bastante inferior a la demanda de transporte; esto ha traído como consecuencia, incrementos en la congestión, demoras y problemas ambientales.

Para solucionar los problemas de intersecciones viales, los diseños geométricos se deben trabajar no sólo en función del radio de la curva, sino en función de otros elementos como la tangente, la externa, el disloque o puntos obligados; considerando las limitaciones que existen debido al espacio disponible.

Además de las implicaciones matemáticas que pueda generar un diseño, los impactos sobre la sociedad pocas veces se tienen en cuenta. Hasta hace pocos años se ha intentado involucrar el factor humano y ambiental a un diseño en el campo de las vías.

Esta situación plantea la necesidad de diseñar una infraestructura vial que optimice las exigencias presentadas por la circulación vehicular, teniendo como objetivo principal proporcionar un diseño eficiente, seguro, económico y que esté acorde con los recursos disponibles que cada vez resultan más limitados en la medida que el crecimiento de la construcción densifica la ciudad al punto de hacer crítica la disposición de espacio destinado para el transporte.

Es indiscutible que el ingenio y creación de estas obras están llevados al máximo reto; a alcanzar niveles de mallas viales de países no mas desarrollados con presupuestos representativamente menores. Mientras en Colombia se destina alrededor de un $0.8 \%$ del presupuesto de la nación, para el desarrollo vial, en otros países este porcentaje llega a ser del orden del $2 \%$ al $3 \%$. Aún así, estas cifras, no desconocen la edificación de grandes obras de Ingeniería como el Viaducto Cesar Gaviria en la Ciudad de Pereira o la intersección de la calle 93 con Autopista Norte en Bogotá.

En este panorama, y según la divulgación hecha en "Caracol Radio", en el año 2010 se reflejará la inversión de 718 mil millones de pesos en diseños y construcción de vías en Bogotá dentro de las cuales se contempla la construcción de 6 intersecciones.

\section{Marco teórico}

Hasta 1972 se desarrollaban diseños de vías sin criterios definidos. -El Ministerio de Obras Públicas editó en ese año las primeras Normas de Diseño Geométrico; pero éstas eran "empíricas" y únicamente solucionaban curvas simples y compuestas pero sin tener bases científicas ni matemáticas-. Estas normas cum- 
plían su función para ese momento, contando con que las velocidades de diseño no eran mayores de $50 \mathrm{~km} / \mathrm{h}$ (Normas de diseño 1972). Años después y con la evolución de los automóviles, se desarrollaron velocidades mayores y se presentó entonces la necesidad de modernizar las vías y por supuesto, como consecuencia, elaborar nuevas normas. El cambio ocurrió en 1998, cuando INVIAS publica las nuevas Normas de Diseño, resultado de una investigación realizada por un grupo de profesionales especializados en el área.

Estas normas determinan a través de cuadros, tablas, notas y gráficos, los parámetros que deben cumplirse en un diseño, pero no desarrollan ecuaciones, ni modelos que faciliten la interpretación pedagógica; convirtiéndolas sólo en material de consulta.

\section{Justificación}

Existen dos factores importantes que determinan la necesidad de intersecciones viales; uno de éstos es la evidencia física de la congestión de tránsito, que en la actualidad muestra puntos críticos y se convierte en prioridad. La otra, es el resultado de la proyección del flujo que arroja un aumento para los próximos años.

Aunado a lo anterior y debido a que la infraestructura suburbana con el tiempo se incorpora a la urbana, es importante, además de estratégico considerar la posibilidad de trabajar en conjunto para asegurar un control de accesos adecuado y así, lograr una planeación integral.

De otra parte, en cuanto a la Seguridad Vial, la tasa de accidentalidad ha aumentado no sólo en el número de accidentes sino también el en el número de vehículos implicados, aumentando también, los índices de mortalidad. Con el objeto de reducir estos índices en Bogotá, y en general en el país, se han tomado medidas preventivas como son: la iluminación, la señalización, control de alcoholemia, el uso del cinturón de seguridad, límites de velocidad, uso del casco para motociclistas y la luz encendida en carretera a cualquier hora del día, entre otras. También se han generado campañas publicitarias como las "Estrellas Negras" debido a que en el área de la infraestructura vial, el presupuesto no permite la implementación de otras alternativas.

\section{Propuesta}

Uno de los propósitos de la temática expuesta en el presente articulo, es que sus contenidos conduzcan a la elaboración de un manual para el diseño de vías urbanas, especialmente intersecciones, teniendo en cuenta las normas de diseño.
Sobre esta idea, y para solucionar cualquier caso de intersección vial, se deben considerar los siguientes pasos:

Identificación del problema: Consiste en el estudio de puntos críticos de la malla vial, aquellos que tienen un alto índice de accidentalidad, represamiento de flujo vehicular, pasos obligados como canales, vías férreas, intersecciones con vías existentes o vías nuevas, etc.

Recopilación de información: Conteos, encuestas, aforos, información existente en las entidades correspondientes sobre el flujo vehicular de la zona. Es uno de los aspectos esenciales en este tipo de estudios, ya que permite ver de una manera mas clara y objetiva los problemas a solucionar. Este proceso debe tener en cuenta los datos que se van a tomar, ¿cuándo?, ¿dónde?, ¿cómo se van analizar? ¿Para qué se van a utilizar? y por supuesto las necesidades que se pretenden suplir.

Planeación: con base en la información obtenida, se determina la vía que tiene prioridad para ofrecerle la mayor comodidad. La planeación también debe considerar el impacto que esto pueda tener sobre el resto de la malla, el impacto ambiental y social sobre las construcciones existentes es fundamental en la toma de decisiones ya que constituye una de las fases de mayor incidencia en el futuro proyecto. Una buena planeación se refleja en la armonía y funcionalidad de la vía.

La problemática ambiental ligada al transporte afecta el aire, el agua, los suelos y la calidad de vida de los habitantes. Desde la perspectiva ambiental, en los últimos años se ha generado, a escala nacional e internacional, un incremento en la conciencia sobre la importancia ecológica, involucrándola en la concepción y fase de estudio de los proyectos en donde anteriormente no se consideraba. En este sentido, y aunque la interacción de la ciudad con las infraestructuras del transporte es necesaria para su desarrollo, es innegable que propicia diversos efectos nocivos dentro del escenario urbano y fuera de él. Con el exceso de contaminación en las ciudades, los impactos ambientales comienzan a tomar la importancia que merecen. Estos impactos no sólo se relacionan con la contaminación del aire; también implica la contaminación por ruido, el deterioro del paisaje urbano y el detrimento en la libre movilización de las personas.

Esto hace necesario que en esta etapa de planeación se deba recopilar la información sobre el monitoreo de estos factores, estudiarlos y plantear medi- 
das para mitigarlos. Los parámetros que se deberán tener en cuenta en el momento de desarrollar un proyecto vial urbano son:

-Ecológicos: el respeto por las condiciones naturales y ambientales preexistentes, cuando ellas lo ameriten. Proponer nuevos y específicos aportes para el mejoramiento ambiental y paisajístico del entorno del proyecto.

- Técnicos o físicos: Referidos a la funcionalidad y habitabilidad de los espacios. Entre los datos físicos el más importante es la topografía del terreno ya que ésta puede utilizarse como ventaja en algunos casos sobretodo en el diseño vertical del proyecto y en otros casos puede representar una restricción. Otro dato físico que es relevante en el diseño de una intersección es el estudio de suelos, las características geológicas y geotécnicas que limiten la dimensión de la intersección, además del espacio físico disponible para la amplitud de las curvas y por último el manejo estratégico del drenaje contando con las redes disponibles.

En Bogotá es evidente la falta de planeación técnica en puntos como la calle 68 por carrera 40 en donde la altura de la rampa del puente que baja hacia el costado occidental no permite que la carrera 40 atraviese por debajo en línea recta obligando al usuario a hacer una curva para esquivar la columna del puente y a reducir de manera brusca la velocidad ya que es una curva muy cerrada.

Aunque las diferentes administraciones han venido solucionando los problemas, en algunas intersecciones viales como la Avenida Jiménez por NQS ó Avenida Jiménez por calle 68, entre otras, construyeron puentes que no fueron parte de una planeación inicial, sigue evidenciándose la ausencia de una mentalidad estratégica, proyectista o futurista que impida que esto siga sucediendo.

-Sociales: Teniendo en cuenta tanto al individuo, como a los diversos grupos que se verán afectados de algún modo por el nuevo proyecto.

Se debe caracterizar la zona afectada, la importancia y movimiento de los corredores peatonales que varía dependiendo de la presencia de centros comerciales, colegios, hospitales, centros deportivos, culturales $\mathrm{O}$ industriales, y la existencia de puentes peatonales o zonas como los pasos "cebra", que faciliten el flujo peatonal.

Se debe considerar también la privacidad en las zonas residenciales ya que puede verse afectada por la altura de los corredores elevados, sea por la contaminación, la visual o por el ruido.

La rodadura de un vehículo, las explosiones de carburante y las vibraciones producidas por los motores constituyen una importante fuente de ruido en las ciudades. El conocimiento de este problema global producido por los vehículos en la ciudad es una premisa básica para tomar decisiones en el diseño de las vías urbanas.

Existen numerosos métodos para el cálculo teórico de los niveles de ruido producido por el tráfico en una carretera desarrollados principalmente en Francia, Estados Unidos, Alemania e Inglaterra, cuyo resultado arroja la estimación de este nivel en las fachadas colindantes a las Vías. La mayor parte de los modelos de medición obtienen como resultado los niveles a cierta distancia y altura del borde de la calzada. Se intenta con ello simular los efectos en los peatones y sobre las viviendas ubicadas en las márgenes de la carretera. Los efectos perjudiciales derivados de los altos niveles de ruido, al que pueden estar expuestos los ciudadanos, obligan a establecer limitaciones que pueden hacer referencia por un lado, al nivel de emisión de la fuente, en este caso el vehículo. Estos niveles pueden obtenerse de dos maneras: por un lado, con modelos teóricos que permiten pronosticar niveles de ruido en función de datos de tráfico y las características geométricas (en el caso de vías nuevas); por otro lado, en las vías existentes será necesario realizar mediciones sistemáticas en puntos aleatorios que permitan obtener valores reales y la verificación de éstos con aproximaciones teóricas.

La contaminación por los olores característicos del tránsito afecta a los ciudadanos de manera diferente incluyendo nauseas, pérdida del apetito y varios efectos subjetivos. Para reducir los impactos en la calidad del aire por fuentes móviles, se debe introducir un programa de control de las emisiones por fuentes móviles, como el que se ha venido ejerciendo con la emisión toxica a través del "Análisis de Gases".

\section{Prediseño}

Como resultado de la planeación, se hacen los bosquejos de las posibles soluciones: Glorietas, intersecciones a nivel, intersecciones a desnivel teniendo en cuenta las prioridades de las bifurcaciones. Aunque no se trata de un diseño geométrico, le da al diseñador una visión general de la solución del problema. Es importante hacer un diagnóstico del funcionamiento de cada una de las soluciones apoyado con programas de simulación. Sin embargo éstos también deberán ponerse a prueba bajo parámetros de análisis social, económico y ambiental. 
A nivel local y más específicamente en una intersección urbana, el problema de tránsito se debe analizar de una manera secuencial: primero estudiar la solución como intersección no semaforizada, posteriormente como glorieta, luego como intersección semaforizada y finalmente como intersección a desnivel. Se recomienda que el movimiento principal de cruce en un enlace se resuelva en el nivel inferior ya que de esta manera disminuye el impacto visual y sonoro del tráfico, además da lugar a menores dimensiones y costos en las estructuras elevadas que se requieran.

\section{Parámetros a tener en cuenta:}

-En el caso de una vía urbana, los estudios sobre velocidad puntual pueden tener varias aplicaciones en el diseño, son necesarios para la determinación del valor de variables para la regulación del tránsito, el análisis de capacidad vial y nivel de servicio, las evaluaciones sobre seguridad vial y estimación de tendencias de velocidades.

Otro dato importante para diseñar es la clasificación de las vías, sean estas tanto vías rurales como urbanas. En este momento se hace necesario conocer la jerarquización de las vías existentes, si es troncal, autopista, vía arteria, principal, secundaria, colectora, local, privada, etc. y así determinar una solución sin impactar negativamente la red vial general. La clasificación dispuesta en el POT (Plan de Ordenamiento Territorial), decreto 619 de Julio 28 de 2000, en el Artículo 158, determina las secciones viales y tiene como base, entre otros, los anchos mínimos para desarrollar las vías.

-El diseñador también debe conocer el estado de los elementos existentes y los pronósticos de planificación relacionados con la vía, el sector y los servicios públicos ya que éstos son determinantes en las características de la vía para plantear el proyecto a un nivel macro y no local.

-El uso del suelo es otro aspecto muy importante y pocas veces incide en la planeación de una vía, es necesario contar con la organización de las zonas urbanas que se está afectando con el diseño, si es una zona industrial, residencial, comercial, etc. tener en cuenta la presencia de hospitales, colegios, o lugares que requieran de especial cuidado en cuanto al ruido que definitivamente generan los vehículos.

- Dentro de los datos funcionales es importante resaltar la clasificación de las vías a intervenir o diseñar ya que la prioridad que tiene el corredor de mayor importancia infiere en el diseño geométrico y económico de la intersección. (Los puentes y depresiones son más costosos y por esto se deben plantear para las vías mas pequeñas).
Para el diseño y mejoramiento de las intersecciones a nivel se debe reducir el número de puntos conflictivos en los movimientos vehiculares además de controlar la velocidad tanto de los vehículos que entran como la de los que salen de la intersección utilizando dispositivos de control como señales o semáforos dependiendo de los volúmenes que maneje la vía.

-En las intersecciones debe existir una visibilidad continua a lo largo de los caminos que se cruzan para permitir a los conductores que se acercan simultáneamente, verse entre sí con la anticipación necesaria.

\section{Diseño}

Como resultado de las anteriores etapas, se puede determinar una serie de puntos obligados que se convertirán en datos de entrada para el cálculo matemático de las curvas. Entonces el diseño consistirá en calcular todos lo elementos geométricos que cumplan con las especificaciones técnicas, normas de diseño y al mismo tiempo sea armonioso con el entorno.

\section{En esta etapa se debe tener en cuenta:}

-Evitar maniobras múltiples y compuestas de convergencia y divergencia ya que crean indecisión por parte del conductor y por consiguiente conflictos adicionales.

- Crear espacios amplios con distancias considerables de visibilidad y con alta capacidad de maniobra favoreciendo las vías que manejan los flujos más fuertes o más rápidos, y así minimizar peligros y demoras.

- Consultar las normas de diseño en los ítems relacionados con: Distancias de visibilidad de parada y de adelantamiento.

-En cuanto al diseño vertical se debe tener en cuenta parámetros como alturas y pendientes reglamentarias, necesarias para las intersecciones a desnivel.

-Los espacios necesarios para los cruces peatonales.

Conocer todas las alternativas disponibles en el cálculo de curvas para elaborar la mejor solución. Existen diversas combinaciones de curvas horizontales y verticales que el diseñador puede tener como herramienta para cumplir con este objetivo. 


\section{Clasificación de las curvas horizontales}

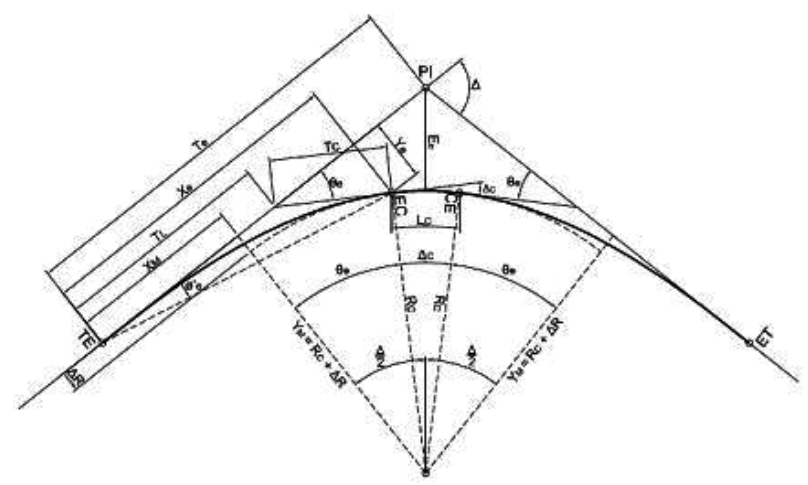

Figura 4. Curva Espiral Circular Espiral. Fuente: El autor

\section{Curva Espiral Circular Espiral:}

Este es el caso más general, consiste en calcular los elementos en función del Radio que es el único elemento que suponemos. Se presenta cuándo no se encuentran en el proceso problemas con la topografía o con las normas de diseño.

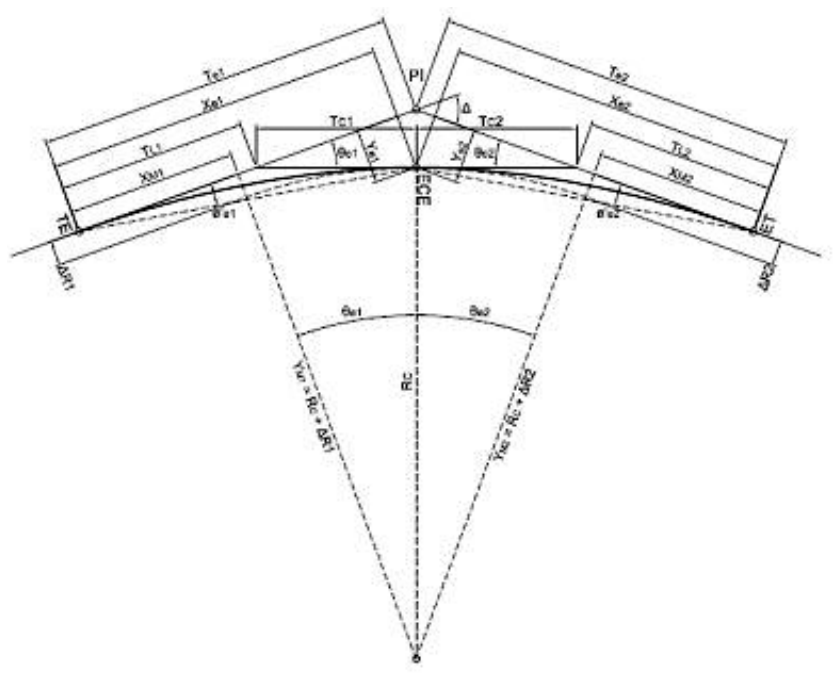

Figura 5. Curva Espiral - Espiral. Fuente: El autor

\section{Curva Espiral - Espiral:}

Estas curvas aparecen en el diseño cuando la longitud de la circular es menor o igual al arco unitario y/o menor o igual a 1 segundo la velocidad de diseño ó cuándo la delta de diseño es menor de $20^{\circ}$ para cualquier velocidad de Diseño.

El diseñador en lo posible debe evitar estas curvas, aunque la norma en ocasiones las permita con el único fin de evitar las Espiral -Circular -Espiral mal diseñadas, pero no es recomendable ya que no da lugar a la transición del peralte.
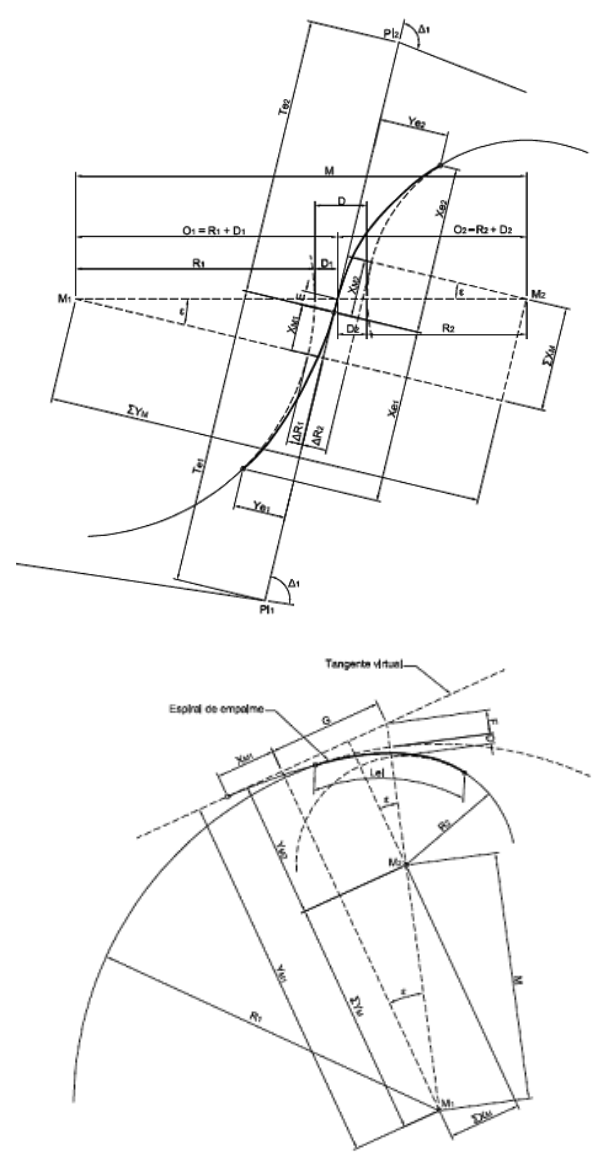

Figuras 6 y 7. Espiral en "S" y en "C". Fuente: El autor

\section{Espiral en "S" $y$ en "C":}

Estas son las curvas que no tienen entre tangencia. La espiral en "S" es muy común mientras la espiral en "C" es mejor remplazarla por una espiral asimétrica. Cuando se hace necesario diseñar una curva en "S" por un cambio brusco de dirección la Norma de Diseño la acepta con la condición de que exista un punto de inflexión. Estas curvas se presentan cuando la poligonal de diseño, tiene los PI (puntos de intersección) muy cercanos y no se pueden mover.

Espiral para $\Delta=180^{\circ}$

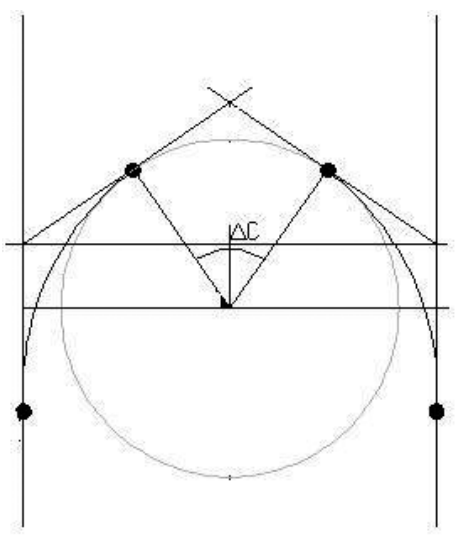

Figura 8. Dibujo Curva entre Paralelas. Fuente: El autor 
Su principal aplicación es hacer retornos en avenidas

\section{Espiral para $\Delta>180^{\circ}$ (Espiral en bombillo):}

Comúnmente, esta curva es utilizada para las orejas de los puentes en las intersecciones viales. Es práctico calcular en función de la externa porque el diseñador determina el tamaño de la oreja.

\section{Criterios para el diseño vertical}

La parábola cuadrática o de segundo grado, es la curva ideal que une alineamientos verticales.

Cuando se habla de la longitud de la curva vertical se refiere a su proyección horizontal. En lo posible y sólo por facilidad el diseñador puede redondear esta cifra a números múltiplos de 20 con el fin de agilizar los cálculos de las cotas de cada abscisa.

En el momento de hacer el trazado de los alineamientos verticales es recomendable buscar cambios graduales de pendientes, trazar alineamientos largos respetando la longitud mínima entre Pls verticales que indica la norma según la velocidad de diseño.

En tramos de acceso, suavizar la pendiente en la cima y tener en cuenta que en bifurcaciones no se recomiendan pendientes mayores al $4 \%$.

Para un buen trazado las pendientes mayores podrían plantearse en el tramo inicial del ascenso y las pendientes menores en la parte superior. Sean curvas convexas o cóncavas, se trata de hacer la curva lo mas larga posible con el fin de evitar problemas de visibilidad.

\section{Tipos de Intersecciones}

Las intersecciones tienen una clasificación que va desde la más simple a la más compleja y es necesario analizarlas para la toma de decisiones. En orden de importancia son: Intersecciones a nivel simples, Intersecciones a nivel con carriles adicionales para cambios de velocidad, Intersecciones canalizadas, Glorietas e Intersecciones a desnivel.

El diseño de intersecciones a desnivel depende de factores de tránsito como volúmenes horarios, velocidad del proyecto y por lo general se diseñan cuando las intersecciones a nivel no tienen la capacidad suficiente para ofrecer los movimientos planeados.

Es importante diseñar estratégicamente los accesos ya que los ramales de un enlace tienen que adaptar su velocidad de salida a las condiciones de las vías de entrada. En el caso de tener limitaciones en cuanto a las longitudes de estos ramales es necesario el diseño de dispositivos de control que adviertan al conductor sobre los cambios bruscos de velocidad.

En el caso de las Glorietas el factor cultural tiene mucha incidencia ya que por lo general su funcionamiento se basa en el respeto a la vía que tiene los vehículos que están en el interior, dando espera a los que van a ingresar al anillo vial que comunica las vías que intervienen.

Las numerosas ventajas que ofrecen las glorietas, tales como permitir un movimiento continuo y ordenado del tránsito, disminuir conflictos entre vehículos al eliminar los cruces, sobre todo en cuanto a seguridad, han llevado a los ingenieros a multiplicar este tipo de solución. La principal ventaja de una glorieta es que normalmente cuesta menos que un cruce a desnivel semaforizado, que pudiera construirse en la misma área. Además, la circulación en un solo sentido dentro de la glorieta ofrece un movimiento continuo y ordenado cuando se opera a bajos volúmenes de tránsito y son especialmente adecuadas para intersecciones de 5 o más accesos. Sin embargo, las glorietas son desventajosas en zonas que tienen restricción de espacio y se acomodan a terrenos generalmente planos. Además tienden a saturarse cuándo más de dos de las vías involucradas se aproximan a su capacidad. En algunos casos, en zonas urbanas, las glorietas operan mediante semáforos, lo que contradice el principio básico de las glorietas que es la circulación continua.

\section{Ciclorutas}

Debido a la problemática que actualmente se evidencia con la congestión del transporte y los impactos ambientales producidos por la operación vehicular, en los últimos años se ha venido reconociendo a la bicicleta, como un modo viable de transporte.

Las ciclorutas, están localizadas a los lados de algunas vías principales, intermedias y a través de los parques, a lo largo de gran parte de la ciudad con el propósito de fomentar el uso de este modo alternativo de transporte y como consecuencia directa, ayudar a bajar el nivel de contaminación. Es importante entonces como diseñadores involucrar en este proceso el espacio necesario para el desarrollo de estos recursos.

Generalmente, una cicloruta beneficia todo el sistema incluyendo a los otros modos de transporte y su implementación debe estar en armonía con los objetivos generales de la comunidad. Un programa de ciclo vías debe considerar, además del diseño y construcción de la infraestructura, el factor cultural por medio de educación, disposición de personal 
entrenado, incentivos para el uso de la bicicleta y la definición de normas claras al respecto.

\section{Drenajes}

El drenaje es el componente del diseño destinado a proteger la estructura de la acción destructiva del agua. Resulta normalmente más difícil y costoso en las carreteras urbanas, debido al daño potencial que pueden producir los volúmenes de agua y la dificultad en la construcción de sistemas de evacuación de aguas superficiales, como cunetas, en zonas de cruces de peatones. El objetivo final de involucrar estas obras en un diseño es reducir los costos que generan tanto la construcción como el mantenimiento de una vía.

El diseñador geométrico finalmente aporta con este aspecto tan importante, al calcular las pendientes longitudinales y transversales. La pendiente transversal recomendada en calzadas urbanas está entre el $2 \%$ y el $3 \%$, dependiendo del tipo de pavimento (flexible, rígido, articulado) y la calidad de toda la infraestructura en general.

Las cunetas se deben diseñar considerando que la pendiente longitudinal no propicie el apozamiento de agua. Para obtener un adecuado funcionamiento, las cunetas deben garantizar una pendiente mínima del $0.3 \%$. Para evacuar rápidamente el agua lluvia que corre por la superficie del pavimento y por las cunetas, es necesario construir sumideros que consisten en aberturas que se disponen en las cunetas para recibir el agua y entregarla a una tubería de conducción que la lleve a la red del alcantarillado, generalmente a través de un pozo de inspección colocado en el cruce de dos calles, mientras el uso de terrazas, la vegetación y otros mecanismos de control de drenaje pueden reducir la erosión y permitir que el agua recargue las reservas del nivel freático.

\section{Señalización}

Los dispositivos para el control de tránsito son las señales, semáforos y cualquier otro que se ubique estratégicamente con el propósito de prevenir, regular y guiar a los usuarios sobre la manera correcta y segura de circulación. Estos previenen a los conductores y peatones sobre las limitaciones o restricciones que existen en el sistema.

Es recomendable emplear sólo señales precisas y estratégicamente ubicadas para conseguir una mejor atención. En áreas urbanas, evitar la confusión con letreros de publicidad, la interferencia de vegetación o la deficiencia de visibilidad nocturna por contrastes de luz.
El factor importante a tener en cuenta entre las señales de pare y ceda el paso, para efectos de instalación, es la visibilidad del cruce, por lo tanto debe analizarse el triángulo de visibilidad, y si la intersección cumple con este requisito se procederá a la instalación de la señal de ceda el paso, ya que el conductor que circula por la vía secundaría puede identificar si transita otro vehículo por la principal, para reaccionar y detener el auto, en caso contrario se requiere la señal de pare.

\section{Iluminación}

La iluminación para una intersección vial debe contemplarse, no solamente desde una perspectiva funcional sino también estética. La instalación de luminarias en las vías debe ser acorde a la escala y dimensión del proyecto y el entorno.

Es aconsejable que la vía esté generosamente iluminada cuándo existan altas intensidades de tráfico y sobretodo si existen varias intersecciones en un tramo de vía urbana. En este caso, puede recomendarse la iluminación adicional en las vías que las conecten.

Es de especial cuidado la iluminación en los puntos donde existan cambios en la sección transversal que obliguen a variaciones de velocidad en los vehículos, donde la relación entre accidentes nocturnos y diurnos es considerable, en intersecciones urbanas y túneles.

Las intersecciones urbanas deberán tener mejor iluminación que el resto de las calles, debido al posible incremento de la velocidad durante la noche buscando la mayor visibilidad posible entre peatones y conductores.

Es importante evitar áreas de fuerte contraste luminoso, tales como entradas y salidas de túneles o deprimidos.

\section{Conclusión}

-El presente artículo es una propuesta donde se establecen de manera secuencial, los criterios y parámetros para obtener la solución más viable, más económica y sobretodo más funcional a un problema vial en las redes urbanas. Es responsabilidad, entonces, del diseñador geométrico calcular las intersecciones viales tomando como dato de entrada el resultado de todo un estudio previo que involucre el factor social, ambiental y económico, para que apoyado por una gran diversidad de alternativas matemáticas, satisfaga integralmente los requerimientos de este trabajo. 


\author{
X. Referencias \\ [1] Estado del arte- Secretaria de Tránsito y transporte \\ [2] Normas IDU \\ [3] Normas INVIAS 1972 \\ [4] Normas INVIAS 1978 \\ [5] Normas INVIAS 1998
}

Sandra Liliana Uribe C. Ingeniera civil de la Universidad De La Salle. Magister en Ingeniería Civil énfasis en Infraestructura Vial; integrante del grupo GEIIC.MD; docente del área de vías, programa de Ingeniería Civil de UNIMINUTO.

chana511@gmail.com 